\title{
A wireless portable physiology recorder for psychophysiology research based on a personal digital assistant
}

\author{
THIJS SCHRAMA \\ Leiden University, Leiden, The Netherlands
}

\begin{abstract}
Psychophysiology research is increasingly relying on portable instruments that can assess physiological responses during real-life situations at locations outside of research labs, such as at school, home, work, and outdoors. In this article, I report on the feasibility of a personal digital assistant-based portable physiology recording system with online signal graphing and wireless digital telemetry for psychophysiology research. I demonstrate that such a system can measure electrocardiogram and electrodermal activity and send this data over a wireless communication link to a PC. It enables users to inspect the integrity of the acquired signals on the portable device and on a PC base station, and it allows users to place time markers for online data analysis.
\end{abstract}

In psychophysiological research, physiological measurements of participants are taken under varying circumstances. For example, physiological responses may be recorded during a memory test, during an emotionally stressful task, when participants are at rest, or even while participants are asleep. Researchers can simultaneously measure various physiological signals such as those in an electrocardiogram (ECG), which records the electrical activity of the heart over time, and electrodermal activity (EDA), the electrical impedance of the skin over time. These signals represent different ways to measure the physiological state of a person, and they can be influenced by levels of attention and arousal. The EDA is associated with activity of the sympathetic nervous system and with emotional arousal, such as fear and anger. If someone is presented with a difficult question, heart rate and breathing rate may go up, and skin resistance may change. Other examples of physiological signals that have been used in behavior and psychological research are thorax impedance and blood pressure.

Practical and ethical concerns limit the types of events and circumstances that can be presented in psychophysiological laboratory settings. Furthermore, to minimize the influence of a measuring device on the human subject's attention during a task or activity, the measurement device must be as nonintrusive as possible. Researchers are therefore relying increasingly on portable instruments that can assess physiological responses during real-life situations at locations outside of research labs, such as at school, home, work, and outdoors (Goodwin, Velicer, \& Intille, 2008). Such portable devices are preferably small and light, and have telemetry functionality. An additional advantage of a portable measurement device is that, because it is battery powered, it is not connected to the high-voltage power grid and therefore provides an inherent measure of safety against electrical faults.

Since most research groups do not have the resources to develop their own portable data-acquisition devices, they often rely on instruments that are commercially available. The number of commercially available portable monitoring instruments has increased during the past few years (Ebner-Priemer \& Kubiak, 2007). Sensors and instruments are now available that exploit the digital, wireless data-communication capabilities that are normally found in computer hardware and personal digital assistants (PDAs) that use technologies such as WiFi and Bluetooth. For an example, see the Porti system from TMS International. A relatively new development is the use of PDAs as portable measurement devices (Held \& Manser, 2005; Lobo et al., 2007; Rodriguez, Goñi, \& Illarramendi, 2005).

Using a PDA as the central part of a physiology recording instrument has some major advantages. A PDA avoids the need for developing a hardware platform. A PDA running on a Windows Mobile operating system avoids low-level microprocessor programming and enables the use of higher-level software development tools. A PDA enables users to give input via a touch screen, while also providing the user output, via the same touch panel, that is much like a graphical user interface (GUI) on ordinary personal computers. A PDA already has data-storage capabilities on its onboard RAM and on its extendable SD memory card. It allows for easy technical upgrades, as the industry regularly offers new PDA models with higher performance levels.

A PDA-based measurement system can exploit the portability, computational, communication, and graphical- 


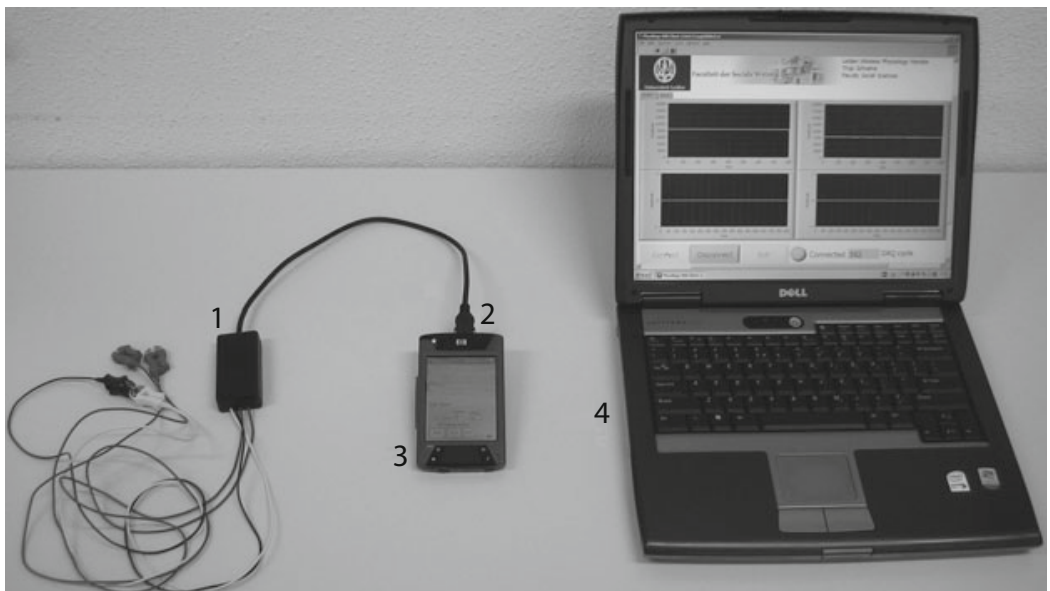

Figure 1. Photo of the complete personal digital assistant (PDA) physiology recording system. The preamplifier (1) is connected to the analog-to-digital converter (2) in the PDA (3), which is running the measurement software, and a laptop (4) is running the telemetry software.

interface capabilities of commercially available PDAs. I therefore decided to investigate the feasibility of a PDAbased portable physiology recording system, for psychophysiology research, with online signal graphing and wireless digital telemetry for high-quality ECG and EDA signals during typical psychological tasks.

\section{METHOD}

\section{Hardware Design}

The complete system consists of (1) a preamplifier, (2) an analogto-digital converter (ADC), (3) a PDA that is running the physiologyrecorder software application, and (4) a PC or laptop that is running the telemetry software application (Figure 1).

I built a small preamplifier $(79 \times 38 \times 19 \mathrm{~mm})$ that conditions the ECG and EDA signals and feeds them to the ADC. The preamplifier is completely encapsulated in plastic and is powered by the PDA. No extra batteries are needed, and the user cannot make adjustments. To maximize the signal quality, special attention was given to the signal conditioning of the EDA and ECG signals. The ECG preamplifier was designed following design principles from Texas Instruments (Texas Instruments, 2004, p. 17). The bandwidth was designed to be $0.5-35 \mathrm{~Hz}(-3 \mathrm{~dB})$, and the gain is $500 \times$, resulting in an input range of -10 to $+10 \mathrm{mV}$. The preamplifier provides active driven cable shields to minimize interference. The EDA preamplifier provides a 500-mV DC-excitation voltage and a low-noise current-to-voltage converter. The input range of the EDA preamplifier is 0-50 microsiemens. EDA signal quality was further improved by massively oversampling the EDA signal at $200 \mathrm{~Hz}$ and by subsequent low-pass filtering, effectively dithering any quantization noise.

The system was developed on a Hewlett-Packard iPAQ hx2750 PDA. The hx2750 ran on the Windows Mobile 2003 Second Edition Operation System. It had an Intel $624 \mathrm{MHz}$ Xscale CPU, $128 \mathrm{MB}$ of RAM, 128 MB of flash memory, a 3.5-in. VGA $320 \times 240$ pixel display, Bluetooth, WiFi $802.11 \mathrm{~b}$ wireless networking, an SD memory card, and a CompactFlash Type II slot. The SD card slot was used for a 2-GB memory card for data storage. The 2-GB SD card provided enough memory for $350 \mathrm{~h}$ of four-channel data.

Analog-to-digital conversion took place in a National Instruments CF6004 data-acquisition card, which was placed in the CompactFlash extension slot and was connected to the preamplifier. This ADC has a maximum sustainable sample rate of $40 \mathrm{~K}$ samples per sec. The bandwidth for ECG and EDA signals can be limited to $100 \mathrm{~Hz}$, so the ADC had enough bandwidth for the ECG and EDA signals that the system was designed to record. In total, four independent channels could be simultaneously measured by the system, although the present system used only two channels.

\section{Software Design}

The PDA physiology recording software application was built with the National Instruments Professional Development Suite and the LabVIEW PDA toolbox, version 7.1. The software was designed to incorporate three continuous loops: one data-producer loop containing all code that handles the ADC communication and GUI, and two data consumer loops. One consumer loop handles the WiFi telemetry, and the second consumer loop handles the data stream for saving to a data file. The data format was kept as simple as possible, using a binary format consisting only of a stream of I16 numbers. Each channel's data are written to a separate file. A new file is automatically created every time a user starts a new data-acquisition session, using the current date, time, and channel number for a file name. Using this very simple binary I16 format, I kept the data stream to 400 bytes per sec per channel.

The data is transferred from the producer loop to the telemetryand file-consumer loops every $250 \mathrm{msec}$ via a data queue. Data is buffered automatically in the data queues. All three loops can therefore function independently, which prevents any lockups or loss of data if one of the consumer loops is lagging or fails. If the telemetry loop or data-saving loop fails, a backlog indicator shows the number of data-acquisition iterations that the loop is lagging. The backlog indicator was designed to count up and down as the number of iterations that the loop is lagging increases or decreases.

The GUI on the PDA was kept as simple as possible, so that users would not be confused by too many settings or choices. PDAs have a small display, so I designed a tabbed GUI (Figure 2). On the first tab, signal traces of the captured data can be directly inspected in a graphical view, and signal integrity can be checked. The first tab also has a start button, stop button, exit button, channel selector, WiFi selector, and WiFi connection-status indicator. The second tab shows the backlog indicator and error indicators. No hardware buttons on the PDA are used. There are no optional user settings except for the WiFi selector.

\section{Telemetry and Online Data Analysis}

The HP iPAQ 2750, like most other PDAs, has a built-in WiFi and Bluetooth adapter that can be used to transfer data to a PC or laptop. Although the bandwidth of Bluetooth connections is suf- 
A

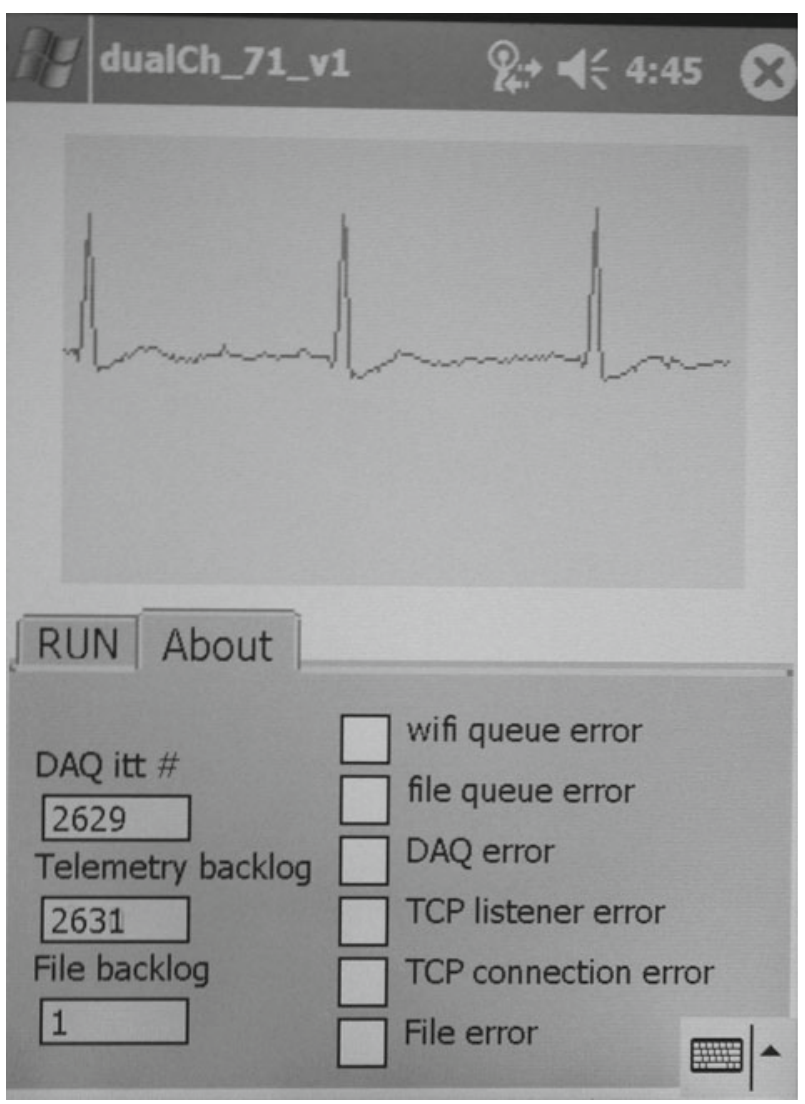

B

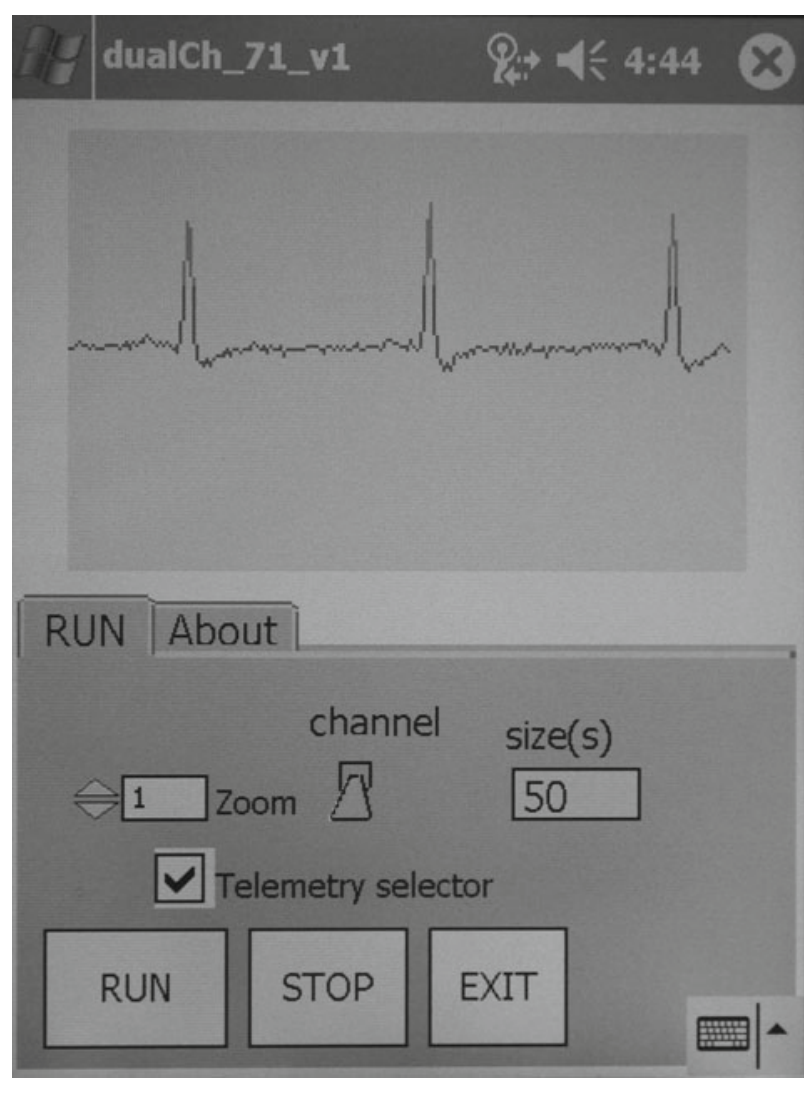

Figure 2. Photos of the two user interface tabs of the personal digital assistant physiology recorder application: online graphing display (A) and software status display (B).

ficient for most psychophysiology signals, its connection range is limited to about $10 \mathrm{~m}$. WiFi communication requires more energy than does Bluetooth communication, resulting in lower battery life, but it extends the connection range to beyond $50 \mathrm{~m}$, which can be advantageous to researchers wishing to let the subject move around freely. I therefore designed a PC-telemetry software application that connects to the PDA via a WiFi TCP/IP network connection. This $\mathrm{PC}$ application displays the signals it receives and saves the data to the local hard drive using a data format identical to that used by the PDA application. Figure 3 shows the GUI of the telemetry application, displaying an online ECG signal. If the connection somehow fails, the data are buffered on the PDA until the WiFi connection is restored. Upon reconnection, all data that have not been successfully sent to the PC previously are sent. In this way, no data are lost as a result of the temporary failure of the WiFi connection. Multiple instances of the PC-telemetry application can be run simultaneously on a single PC, and every instance of the telemetry application can connect to a different PDA. Users are asked to identify the PDA they need to connect to by specifying its IP number. This enables one PC to receive data from multiple PDAs simultaneously.

The PC-telemetry application enables users to set time markers with a mouse click on a marker button. The user can specify a text label for the marker for easy offline interpretation. As a new marker is added, the application calculates the average skin conductance level, the average interbeat interval (IBI), the average heart rate, the number of detected heartbeats, and the root mean square successive difference (RMSSD) of the IBIs in the period between the previous marker and the new marker. When the user ends the recording, the marker times, marker labels, and measurements are stored in a tab-delimited ASCII file. An additional file is created that contains all the IBIs of the whole ECG measurement, to facilitate further offline analysis.

\section{RECORDING EXAMPLES}

Figure 1 shows the complete system, consisting of the preamplifier and electrode cables, a PDA running the physiology recording application, and a laptop running the telemetry application. I made test recordings of ECG and EDA signals. The resulting signals are displayed in Figure 4. The ECG signal shows the typical QRS complexes of an ECG recording. The EDA graph shows that I was able to obtain good quality EDA signals. The average noise level, measured over a period of $1 \mathrm{sec}$, was about 0.001 microsiemens RMS. The system would run for about $135 \mathrm{~min}$ before the batteries needed to be recharged; therefore, the system is suitable mainly for short, task-related psychophysiology research experiments. The PDA application was also tested on two more recent HP iPAQ PDA models, the HP iPAQ hx4700 and the HP iPAQ 214. On both models, the application ran correctly without any modifications to the source code. 
A

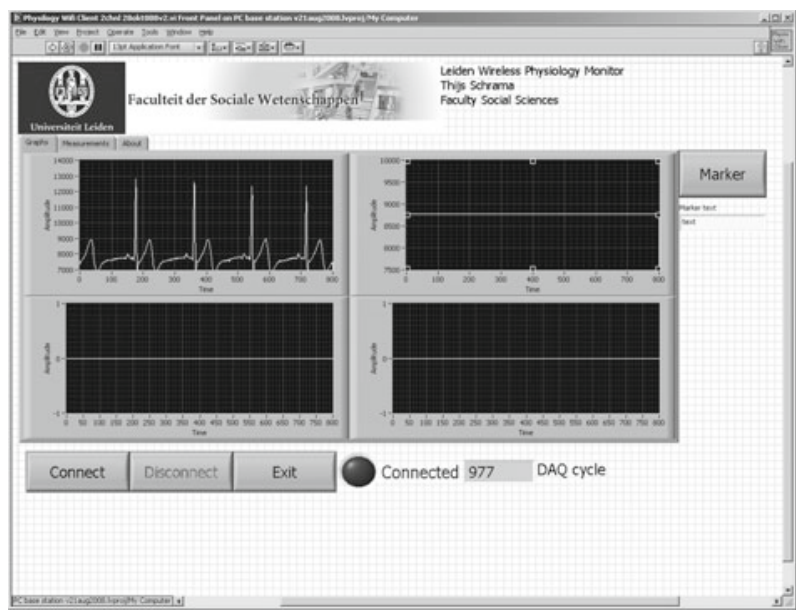

B

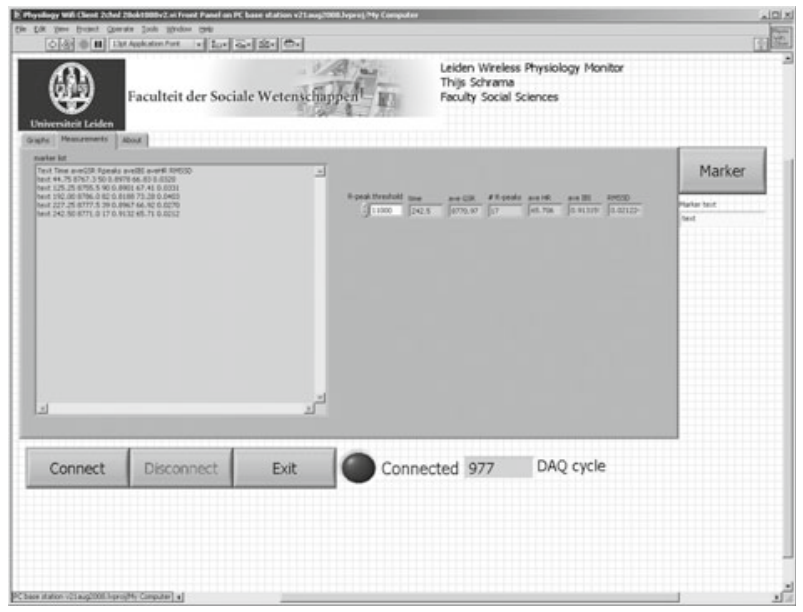

C

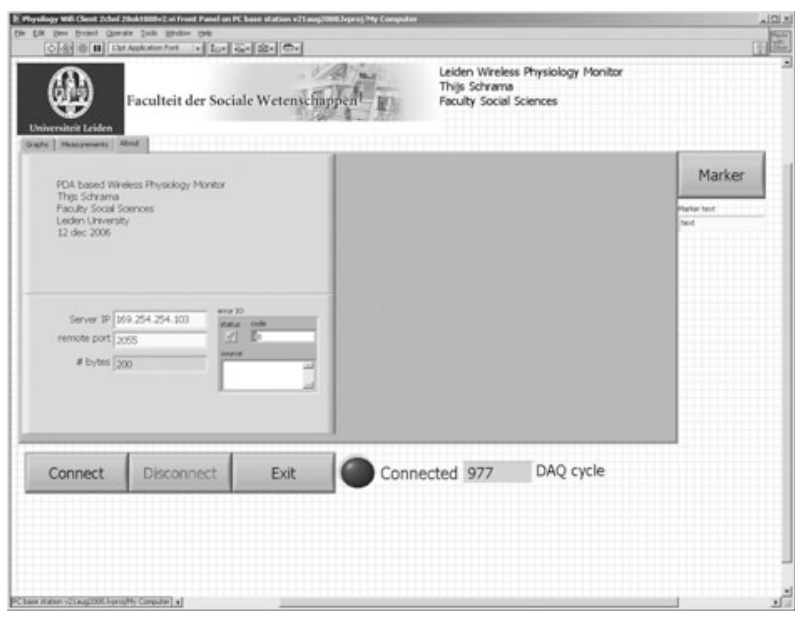

Figure 3. Picture of the three user interface tabs of the telemetry software application GUI: online graphing display (A), event markers and online measurements (B), and network settings $(C)$. 


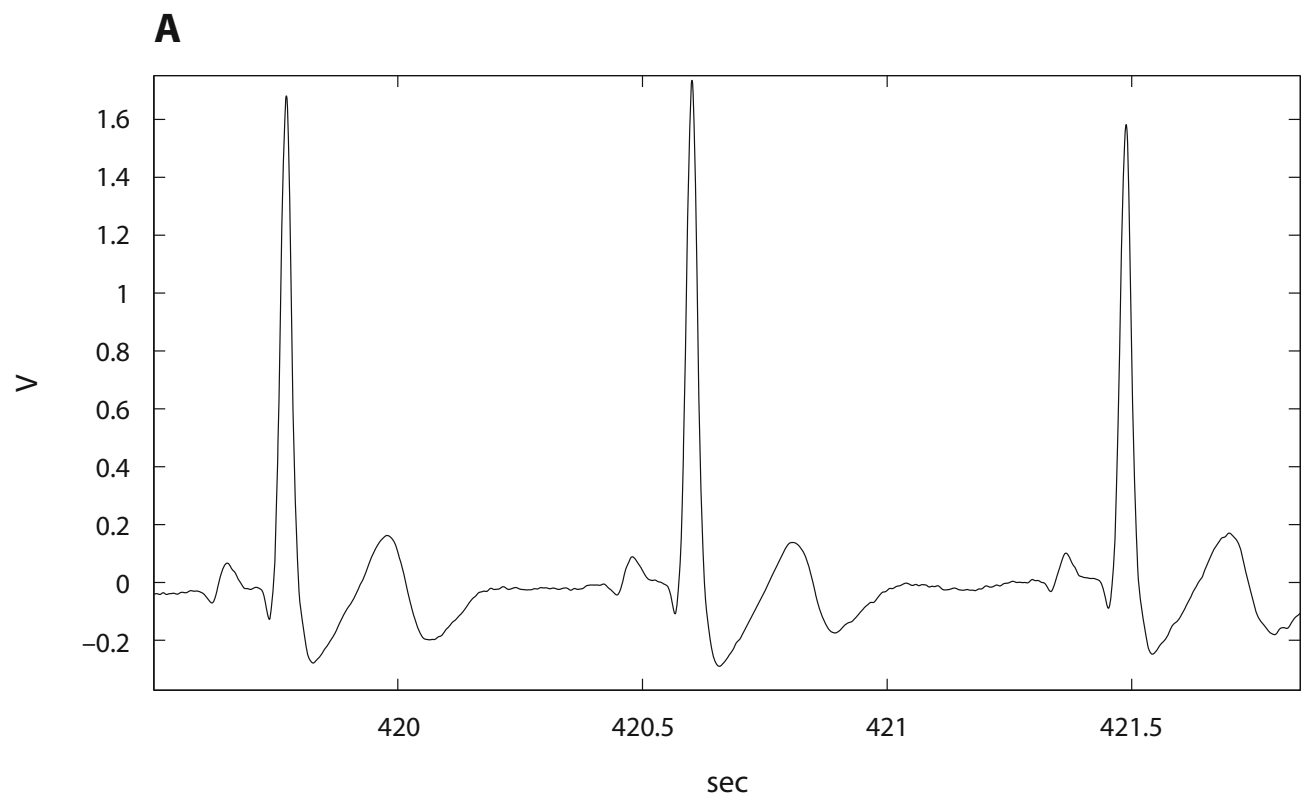

B

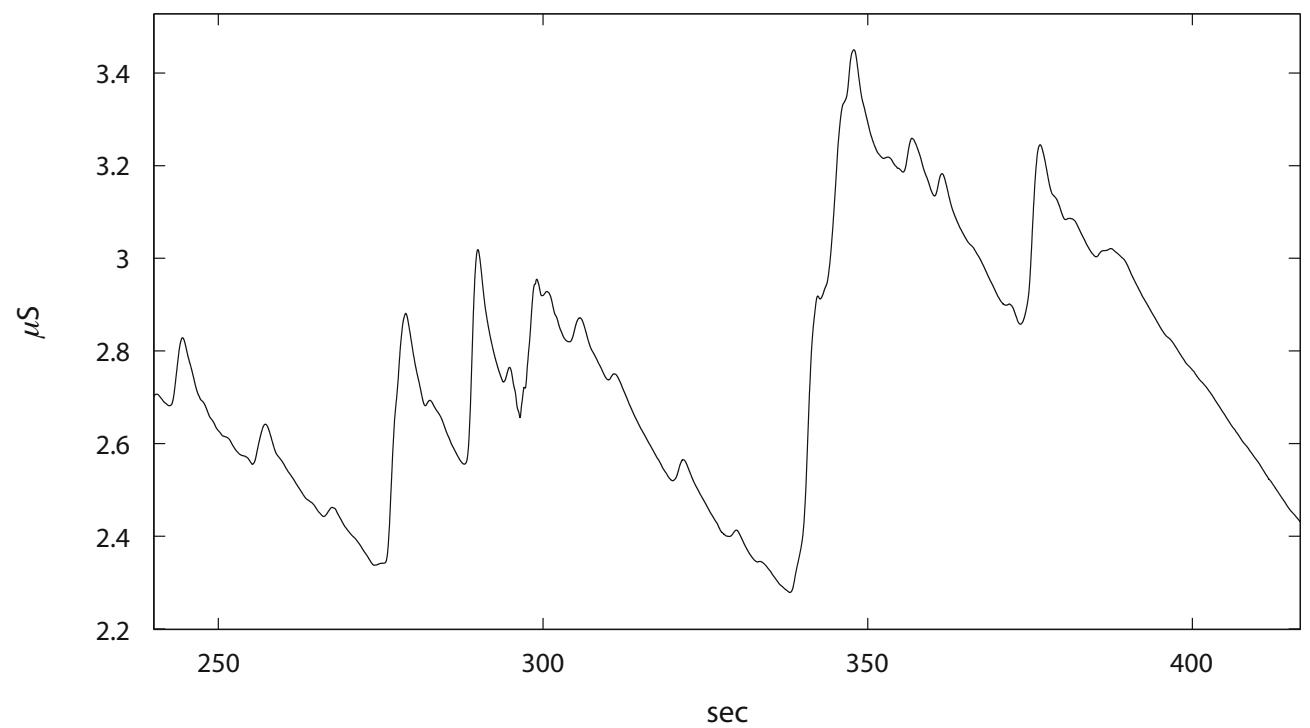

Figure 4. Examples of electrocardiogram (A) and electrodermal activity (B) signals that were acquired with the personal digital assistant physiology-recorder application running on a Hewlett-Packard iPAQ hx2750.

I tested the WiFi telemetry capabilities and found that once the TCP/IP settings were configured correctly, the WiFi network had no problems sending the physiology signal. In an analog-linked telemetry signal, signal corruption and signal-to-noise ratio can be influenced by the connection quality and nearby radio sources. The WiFi telemetry uses a digital network, so no signal degradation could have taken place. The data that were saved on the PC were identical to the data that were saved on the PDA. When I intentionally disconnected the WiFi connection, the system automatically reconnected, and all previous data were sent without data loss.

\section{CONCLUSION AND FUTURE WORK}

This article demonstrates that modern, commercially available PDAs can be deployed successfully as a platform for physiological recordings, especially for relatively low-frequency signals such as the ECG and EDA. Both the sample rate and analog resolution of the device present no signal-quality limitations. The WiFi-based telemetry enables users to check the signal integrity on both the PDA screen and on a PC base station, and it enables online analysis on the PC base station. By storing the entire waveform of the signals on both the PDA 
and the PC base station, it provides an extra safety measure against data loss. The system is portable, wireless, and battery powered; therefore, it is not connected to the $230-\mathrm{V}$ power grid in any way. I have been able to avoid a custom-designed hardware platform and managed to obtain all the necessary functionalities at little cost. Using LabVIEW development software, I avoided low-level programming, and the software can be ported easily to other hardware platforms such as touch panels and ordinary PCs. The limited CPU usage on the PDA (about $12 \%$ ) allows for even more functionality to be added to the PDA software application. Future efforts will be aimed at adding the following: (1) online data analysis on the PDA (e.g., mean heart rate and IBI, RMSSD, and spectral heart-rate variability measures on the PDA), (2) interface to other types of sensors, such as temperature, chest pressure, and thorax impedance sensors, and (3) deployment of the developed software framework on other devices, such as laptops and touch-panel PCs.

\section{AUTHOR NOTE}

I thank M. H. van IJzendoorn and J. F. Dubbeldam for their support. I also thank A. J. W. van der Does and P. M. den Hartog for their support and advice during preparation of the manuscript. Correspondence concerning this article should be addressed to T. Schrama, Faculty of Social
Sciences, Leiden University, Wassenaarseweg 52, 2333AK Leiden, The Netherlands (e-mail: tschrama@fsw.leidenuniv.nl).

\section{REFERENCES}

Ebner-Priemer, U. W., \& KubiaK, T. (2007). Psychological and psychophysiological ambulatory monitoring: A review of hardware and software solutions. European Journal of Psychological Assessment, 23, 214-226.

Goodwin, M. S., Velicer, W. F., \& IntiLle, S. S. (2008). Telemetric monitoring in the behavior sciences. Behavior Research Methods, 40, 328-341. doi:10.3758/BRM.40.1.328

Held, J., \& Manser, T. (2005). A PDA-based system for online recording and analysis of concurrent events in complex behavioral processes. Behavior Research Methods, 37, 155-164.

Lobo, A. P., Loizou, P. C., Kehtarnavaz, N., Torlak, M., Lee, H., Sharma, A., ET AL. (2007). A PDA-based research platform for cochlear implants. Neural Engineering, 2007. CNE '07. 3rd International IEEE/EMBS Conference on Neural Engineering, 28-31.

Rodriguez, J., GoÑI, A., \& ILlarramendi, A. (2005). Real-time classification of ECGs on a PDA. IEEE Transactions on Information Technology in Biomedicine, 9, 23-34.

TEXAS INSTRUMENTS (2004). Information for medical applications: Amplifiers, connectivity, clocks, data converters, digital signal processors, digital temperature sensors, interface, logic, microcontrollers, power management. (Available from Texas Instruments Incorporated, 14950 FAA Boulevard, Fort Worth, TX 76155-9950)

(Manuscript received November 1, 2008; revision accepted for publication February 24, 2009.) 